\title{
Lawinenopfer: Wie viel Zeit bleibt für die Rettung?
}

\begin{abstract}
Werden Lawinenopfer binnen zehn Minuten geborgen, haben sie eine 91\%ige Überlebenschance. Wer länger im Schnee verschüttet bleibt, erstickt oder stirbt an den Folgen eventueller Verletzungen. So sterben in den Alpen jedes Jahr im Durchschnitt 100 Menschen. Die Beschaffenheit des Schnees spielt bei den möglichen Überlebenszeiten eine entscheidende Rolle.
\end{abstract}

- Wissenschaftler der Simon Fraser University im kanadischen Burnaby haben die Daten aller kanadischen Schneelawinenopfer von Oktober 1980 bis September 2005 (301 Fälle) ausgewertet und mit der Schweizer Datenbank (946 Personen) verglichen.

Der Anteil der Überlebenden beider Länder differierte nicht signifikant $(46,2 \%$ [139/301]) versus $46,9 \%$ [444/946]). Signifikante Differenzen $(\mathrm{p}=0,001)$ wurden aber hinsichtlich des Verlaufs der Überlebenskurven zwischen beiden Ländern berechnet. Die kanadische Überlebenskurve fiel steiler

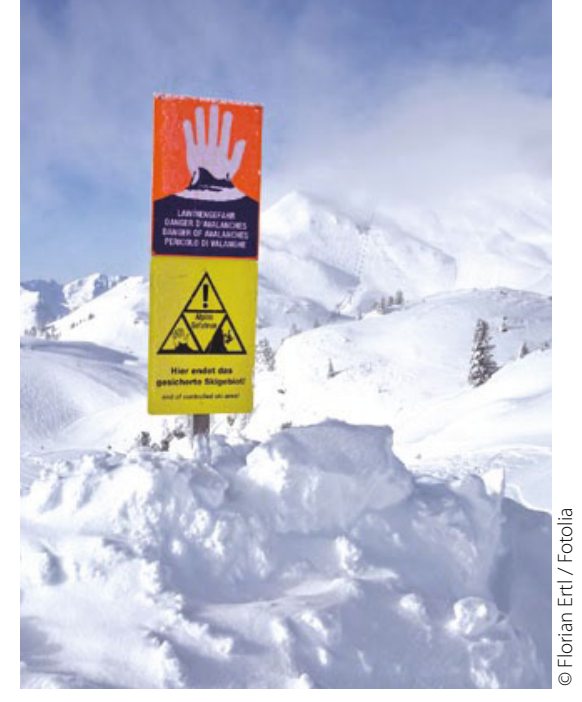

In den Alpen sterben jährlich etwa 100 Menschen in Lawinen.

ab. In manchen Regionen Kanadas ist der Schnee wärmer und deshalb feuchter und dichter gepackt, sodass Lawinenopfer schneller ersticken.

Die Überlebenschancen waren in Kanada vor allem für Traumapatienten schlechter als in der Schweiz, obwohl die Lawinenopfer in Kanada durchschnittlicher schneller geborgen wurden (18 Minuten vs. 35 Minuten). Die schlechteren Ergebnisse sind vermutlich darauf zurückzuführen, dass in Kanada größere Strecken zurückgelegt werden mussten, bis die Lawinenopfer medizinisch versorgt werden konnten.

\section{Kommentar}

Die schnelle Bergung möglichst innerhalb von zehn Minuten bleibt entscheidend für das Überleben von Lawinenopfern. Die Autoren warnen aber auch davor, Wiederbelebungsversuche zu schnell aufzugeben. Bei den tiefen Temperaturen im Schnee kann auch ein längerer Atem- und Herzstillstand u. U. ohne Gehirnschäden überstanden werden.

K. MALBERG =

- P. Haegeli et al.

(School of Resource and Environmental Management, Simon Fraser University, 8888 University Drive, Burnaby, B. C. VSA 156 Canada: E-mail: pascal@avisualanche.ca) Comparison of avalanche survival patterns in Canada and Switzerland. Published on March, 21, 2011 in CMAJ online; doi: 10.1503/cmaj.101435

\section{Schützt ASS auch vor dem Magenkarzinom?}

\section{Dass die regelmäßige Einnahme von ASS vor einem Kolonkarzinom schützen kann, dafür sprechen Er- gebnisse neuerer Untersuchungen. Jetzt wurde im Rahmen einer Studie untersucht, ob dies auch für das Magenkarzinom gilt.}

- Ausgewertet wurden 14 Studien mit insgesamt 5640 Patienten mit einem Magenkarzinom und zwar dahingehend, ob ein Zusammenhang mit der regelmäßigen Einnahme von Aspirin bestand. Das Risiko für ein Nicht-Kardia-Magenkarzinom war bei Patienten unter ASS um rel. 38\% niedriger, und zwar unabhängig davon, ob eine Helico-
bacter-pylori-Infektion vorlag oder nicht.

\section{Kommentar}

Nach den Ergebnissen dieser Studie scheint ASS nicht nur die Manifestation eines Kolon-, sondern auch eines Magenkarzinoms günstig zu beeinflussen. Die Rationale für eine solche chemopräventive Wirkung ist, dass das Enzym Cyclooxygenase, welches durch ASS gehemmt wird, bei der Pathogenese maligner Tumoren beteiligt ist.

Nach neueren Untersuchungen haben $\mathrm{Pa}$ tienten mit einer erhöhten Expression der Cyclooxygenase in der Darmschleimhaut ein erhöhtes Dickdarmkarzinomrisiko. Sicherlich ist es zu früh, eine generelle Chemopräventi- on mit ASS zu propagieren, zumal eine ASSEinnahme ja auch mit gastrointestinalen Komplikationen einhergeht. Bezüglich des Dickdarmkarzinoms empfiehlt sich jedoch schon heute bei Patienten mit einem entfernten Adenom, dieses Gewebe im Hinblick auf die Expression der Cyclooxygenase zu untersuchen. Sollte eine gesteigerte Expression dieses Enzyms nachgewiesen werden, so ist eine Chemoprävention mit ASS durchaus zu erwägen.

P. StiefelHAGen -

\footnotetext{
- P. Yang et al.

Aspirin use and the risk of gastric cancer: A meta-analysis. Dig. Dis. Sci. 55 (2010) 1533-1539
} 